\title{
CARACTERIZAÇÃO DE RESÍDUO SÓLIDO FORMADO EM BIODIESEL DE SEBO BOVINO
}

\author{
Valter José Fernandes Júnior e Antonio de Sousa Araujo \\ Instituto de Química, Universidade Federal do Rio Grande do Norte, Campus Universitário, s/n, 59078-970 Natal - RN, Brasil \\ Fábio da Silva Vinhado e Paulo Roberto Pivesso* \\ Centro de Pesquisas e Análises Tecnológicas, Agência Nacional do Petróleo, Gás Natural e Biocombustíveis, SGAN 603, Módulo \\ H/J, 70830-902 Brasília - DF, Brasil
}

Recebido em 22/6/11; aceito em 25/6/12; publicado na web em 17/9/12

\begin{abstract}
CHARACTERIZATION OF SOLID RESIDUE FORMED IN BEEF TALLOW BIODIESEL. The nature of the solid residue formed in beef tallow biodiesel from two commercial producers in Brazil was determined by comparative analytical techniques, namely, gas chromatography with flame ionization detector (GC-FID) and thermogravimetry (TG). Pure monopalmitin and monostearin were used as reference standards for both methodologies. Analyses were carried out before and after filtration of the solids formed, which allowed the observation that the formation of precipitate reduced the levels of monoglycerides in the beef tallow biodiesel. The chromatographic and thermogravimetric results confirmed the nature of the residue as saturated monoglycerides, predominantly monostearin and monopalmitin.
\end{abstract}

Keywords: monoglycerides; beef tallow; biodiesel.

\section{INTRODUÇÃO}

O biodiesel é um combustível diesel alternativo, composto de monoésteres alquílicos de ácidos graxos derivados de óleos e gorduras de origem vegetal ou animal. ${ }^{1}$ A produção de biodiesel aumentou nos últimos anos em função dos benefícios associados a este combustível, tais como, uso de fontes renováveis, diversidade de matérias-primas, menor toxicidade e biodegradabilidade. Na última década, inovações tecnológicas foram significativas para otimização do processo de transesterificação, com o objetivo de reduzir os níveis de glicerídeos e a produção de glicerina de alta pureza como coproduto, obtendo melhor rendimento e qualidade do biodiesel. ${ }^{2}$ No entanto, tem-se observado que o desempenho do biodiesel em condições de clima frio é claramente pior do que o diesel de petróleo, em função de insolúveis que podem se formar a temperaturas mais baixas. ${ }^{3} \mathrm{~A}$ ocorrência de depósitos em biodiesel pode acelerar o entupimento de filtros de combustíveis e de bicos injetores, causando problemas de desempenho em motores de veículos. ${ }^{4}$ A natureza do precipitado em biodiesel está relacionada ao tipo e pré-tratamento da matéria-prima e à tecnologia de produção do biodiesel.,

Alguns estudos têm-se dedicado à verificação da natureza de resíduos sólidos em biodiesel, relatando que os compostos identificados como esteróis, esteril-ésteres, esteril-glicosídeos e esteril-glicosídeos esterificados são indutores da formação de precipitados em biodiesel de origem vegetal. ${ }^{78}$ Esteróis-glicosídeos esterificados estão presentes em óleos vegetais e durante o processo de transesterificação são convertidos em esteril-glicosídeos, os quais levam à formação de materiais sólidos em biodiesel de origem vegetal e em suas misturas com óleo diesel. ${ }^{9}$ As técnicas de cromatografia a gás e líquida, acopladas a espectrômetros de massas foram aplicadas para se identificar alguns componentes de esteril-glicosídeos em altas concentrações em biodiesel de origem vegetal, sendo observados quatro tipos predominantes, denominados brassicasteril-glicosídeo, estimasteril-glicosídeo, campesteril-glicosídeo e sitosteril-glicosídeo. ${ }^{10,11}$

Com relação à produção de biodiesel no Brasil, é significativo seu crescimento ao longo dos últimos anos. ${ }^{12} \mathrm{Em} 2010$, o Brasil foi o

*e-mail: ppivesso@anp.gov.br segundo maior produtor mundial de biodiesel, atrás da Alemanha. ${ }^{13} \mathrm{~A}$ partir de 2008, a utilização do sebo bovino como matéria-prima para a produção de biodiesel no Brasil tornou-se expressiva, representando cerca de $20 \%$ do biodiesel vendido no país, cuja predominância é biodiesel de soja. ${ }^{14}$

Alguns estudos focando o biodiesel de sebo bovino foram realizados, em sua maioria na investigação sobre as propriedades relacionadas com a estabilidade térmica e oxidativa do biodiesel. Quanto à caracterização do biodiesel de sebo bovino, foi verificado elevado teor de éster saturado em sua composição, relacionado com as altas temperaturas (superiores a $100^{\circ} \mathrm{C}$ ) aplicadas durante a produção. No entanto, não foi descrita qualquer investigação sobre a natureza do resíduo sólido formado. ${ }^{15}$ Segundo dados preliminares obtidos pela Agência Nacional do Petróleo, Gás Natural e Biocombustíveis - ANP, o resíduo sólido de biodiesel de sebo bovino, analisado por cromatografia a gás, mostrou a presença de elevados níveis de ésteres de monoglicerídeos de cadeias saturadas, o que provavelmente ocorre devido a uma cinética de precipitação espontânea e irreversível. ${ }^{16}$ Apesar da ANP estar controlando os níveis de monoglicerídeos no processo de produção de biodiesel, deve-se explorar pesquisas acerca da natureza do resíduo formado e sua influência na qualidade do biodiesel do sebo bovino.

Neste trabalho buscou-se confirmar a composição do precipitado de biodiesel de sebo bovino por meio de diferentes técnicas analíticas de identificação. Inicialmente, foram monitorados os níveis de mono-, di- e triglicerídeos em amostras de biodiesel de sebo bovino de dois produtores comerciais, por período de armazenamento de 12 meses. As amostras foram analisadas pelas técnicas comparativas de cromatografia a gás com detector de ionização de chama (CGDIC) e termogravimetria (TG) para verificar a natureza do resíduo, usando monopalmitina e monoestearina como padrões de referência. Verificou-se que a formação do precipitado reduziu os níveis de monoglicerídeos e aumentou os de di- e triglicerídeos no biodiesel de sebo bovino. Os resultados cromatográficos confirmaram a natureza do resíduo como sendo basicamente monoglicerídeos saturados, com predominância de monopalmitina e monoestearina, este como componente majoritário do precipitado. Além disto, a análise de TG do resíduo indicou perfil semelhante ao de decomposição térmica 
dos padrões de referência, comprovando os resultados anteriores de identificação.

\section{PARTE EXPERIMENTAL}

\section{Coleta e fracionamento do biodiesel de sebo bovino}

Foram coletados $30 \mathrm{~L}$ de biodieseis de sebo bovino (também identificados por B100), recém-produzidos, de dois produtores nacionais autorizados pela ANP que utilizam a rota metílica de transesterificação, identificados como produtores X e Z. As coletas foram realizadas em pontos de amostragem nas áreas de produção, antes de escoar para tanques de armazenamento, para evitar contaminação com lotes anteriores. $\mathrm{O}$ biodiesel de cada produtor foi fracionado em frascos de polietileno de alta densidade de $1 \mathrm{~L}$, com e sem contaminação para simular diferentes condições e tempos de armazenamento.

A Tabela 1 identifica as amostras fracionadas do produtor $\mathrm{X}$. Os códigos numéricos referem-se ao tempo de armazenamento (1 $=1$ mês, $2=2$ meses, $3=3$ meses, $6=6$ meses e $12=12$ meses) e os códigos alfabéticos simulam condições ambientais de tanques de armazenamento, a saber: condição A - amostra a $25^{\circ} \mathrm{C}$ e sem contaminação - simulação de verão; Condição B - amostra com variação de temperatura ( 3 dias $25^{\circ} \mathrm{C}$ e 3 dias $10^{\circ} \mathrm{C}$, alternados) e sem contaminação - simulação de inverno; condição $\mathrm{C}$ - amostra a $25{ }^{\circ} \mathrm{C}$ e contato com resíduo sólido de biodiesel de sebo bovino - simulação de fundo de tanque contaminado com resíduo de biodiesel de sebo bovino de lote anterior; condição D - amostra a $25^{\circ} \mathrm{C}$ e contato com biodiesel de soja - simulação de fundo de tanque contaminado com biodiesel de origem vegetal e condição E - amostra a $25^{\circ} \mathrm{C}$ e contato com pedaço de lã de ferro comercial - simulação de tanque contendo ferro.

Com relação ao fracionamento do biodiesel de sebo bovino do produtor Z, efetuou-se o mesmo procedimento da Tabela 1 , substituindo a letra $\mathrm{X}$ por $\mathrm{Z}$.

\section{Determinação dos teores de mono-, di- e triglicerídeos no biodiesel de sebo bovino}

Para cada mês de armazenamento, foram determinados os teores de monoglicerídeos, diglicerídeos e triglicerídeos no biodiesel por CG-DIC, tanto nas amostras de biodiesel de sebo bovino contendo resíduo sólido como após sua remoção por filtração, com a finalidade de verificar possível variação dos teores de glicerídeos no biodiesel durante a deposição de material sólido.

A análise foi realizada em um cromatógrafo a gás, marca Agilent, modelo $6890 \mathrm{~N}$, de acordo com a norma ASTM D6584. ${ }^{17}$
A identificação dos glicerídeos foi realizada por comparação com os tempos de retenção dos padrões de referência mono-oleína, 1,3 dioleína e trioleína (todos da marca Sigma, 99\% mín. de pureza) utilizados na elaboração da curva analítica, conforme procedimento descrito na norma. Utilizou-se tricaprina como padrão interno para quantificar mono-, di- e triglicerídeos. O método estabelece proceder à silanização (substituição do grupo hidroxila) da amostra com 2,2,2-trifluoro-N-methyl-N-(trimethylsilyl)-acetamide (MSTFA) para obtenção de derivado com menor ponto de ebulição e, assim, permitir melhor eluição dos componentes na coluna cromatográfica. ${ }^{18,19}$

Em um frasco de $20 \mathrm{~mL}$, pesou-se cerca de $100 \mathrm{mg}$ do biodiesel de sebo bovino em balança analítica de resolução $0,1 \mathrm{mg}$. Em seguida, adicionaram-se $100 \mathrm{~mL}$ de butanotriol (Supelco, 99\% mín), $100 \mathrm{~mL}$ de tricaprina (Sigma, 99\% mín) e $100 \mathrm{~mL}$ do reagente derivatizante MSTFA (Merck, grau CG). Após breve agitação da mistura, o frasco foi lacrado e deixou-se reagir por aproximadamente $20 \mathrm{~min}$. Em seguida, o frasco foi aberto e adicionaram-se $8 \mathrm{~mL}$ de $\mathrm{n}$-heptano (Vetec, $99,5 \%$ mín). A solução foi transferida para um frasco de cromatografia gasosa antes da injeção no cromatógrafo, procedendo-se, em seguida, à análise nas condições: injetor cool on column, volume de amostra de $0,5 \mu \mathrm{L}$, temperatura inicial de $50^{\circ} \mathrm{C}$, rate $1: 15^{\circ} \mathrm{C} \mathrm{min}-1$ até $180{ }^{\circ} \mathrm{C}$; rate 2: $7^{\circ} \mathrm{C} \mathrm{min}^{-1}$ até $230{ }^{\circ} \mathrm{C}$, rate $3: 30^{\circ} \mathrm{C} \mathrm{min}^{-1}$ até $380{ }^{\circ} \mathrm{C}$, DIC de $380^{\circ} \mathrm{C}$, gás de arraste $\mathrm{He}$, fluxo de $3 \mathrm{~mL} \mathrm{~min}^{-1}$, coluna capilar de fase estacionária DB-5 (5\%-Phenyl-methylpolysiloxane) com dimensões de $30 \mathrm{~m}$ x $0,25 \mathrm{~mm} \times 0,1 \mu \mathrm{m}$.

Após obtenção do cromatograma, foi calculada a área de cada composto a partir da integração dos picos de mono-, di-, triglicerídeos e tricaprina (padrão interno). A partir da área, da massa da amostra, da massa do padrão interno e dos coeficientes angular e linear da curva analítica de cada componente, calcularam-se as concentrações dos glicerídeos presentes na amostra.

\section{Determinação de monoglicerídeos no resíduo sólido do biodiesel de sebo bovino por CG-DIC}

O resíduo sólido filtrado foi analisado por CG-DIC, aplicando-se a mesma metodologia descrita na norma ASTM D6584; porém, com pequena adaptação acerca da quantidade de amostra pesada (cerca de $2 \mathrm{mg}$ em vez de $100 \mathrm{mg}$ ), por ser resíduo sólido e não biodiesel. Outra adaptação consistiu no uso de piridina (Vetec, 99,5\% mín) para dissolução do resíduo (200 mL).

Foram selecionados resíduos sólidos representativos do início, meio e final do presente estudo, além dos padrões monopalmitina e monoestearina, utilizados para confirmar a presença destes monoglicerídeos nas amostras analisadas: resíduo sólido filtrado das amostras X.1.A, X.3.A e X.12.A (produtor X) e das amostras Z.1.A, Z.3.A e

Tabela 1. Fracionamento do biodiesel de sebo bovino do produtor $\mathrm{X}$

\begin{tabular}{|c|c|c|c|c|c|c|}
\hline \multicolumn{7}{|c|}{ Amostras fracionadas a partir de $30 \mathrm{~L}$ de biodiesel de sebo bovino do produtor $\mathrm{X}$} \\
\hline \multirow{3}{*}{$\begin{array}{l}\text { Condição de } \\
\text { armazenamento }\end{array}$} & \multirow{2}{*}{ Tempo de armazenamento } & Código 1 & Código 2 & Código 3 & Código 6 & Código 12 \\
\hline & & 1 mês & 2 meses & 3 meses & 6 meses & 12 meses \\
\hline & Descrição da condição de armazenamento & \multicolumn{5}{|c|}{ Identificação da amostra fracionada } \\
\hline A & T. ambiente $\left(25^{\circ} \mathrm{C}\right)$ & X.1.A & X.2.A & X.3.A & X.6.A & X.12.A \\
\hline B & 3 dias T. amb. $\left(25^{\circ} \mathrm{C}\right), 3$ dias $10^{\circ} \mathrm{C}$, alternados & X.1.B & X.2.B & X.3.B & X.6.B & X.12.B \\
\hline $\mathrm{C}$ & $\begin{array}{l}\text { T. amb. }\left(25^{\circ} \mathrm{C}\right) \text {, resíduo sólido de biodiesel de } \\
\text { sebo }\left(0,2 \% \text { volume, } 2 \mathrm{~mL} \mathrm{~L}^{-1}\right)\end{array}$ & X.1.C & X.2.C & X.3.C & X.6.C & $\mathrm{X} .12 . \mathrm{C}$ \\
\hline $\mathrm{D}$ & $\begin{array}{l}\text { T. amb. }\left(25^{\circ} \mathrm{C}\right) \text {, biodiesel de soja } \\
\left(1 \% \text { volume }, 10 \mathrm{~mL} \mathrm{~L}^{-1}\right)\end{array}$ & X.1.D & X.2.D & X.3.D & X.6.D & X.12.D \\
\hline E & $\begin{array}{c}\text { T. amb. }\left(25^{\circ} \mathrm{C}\right) \text {, pedaço de lã de ferro } \\
(0,01 \% \text { massa })\end{array}$ & X.1.E & X.2.E & X.3.E & X.6.E & X.12.E \\
\hline
\end{tabular}


Z.12.A (produtor $Z$ ) correspondentes à simulação de verão (condição A) com 1, 3 e 12 meses de armazenamento, respectivamente; padrão de monopalmitina (AccuStandard, código GS-015N, lote 15588) e padrão de monoestearina (AccuStandard, código GS-018N, lote 15391).

\section{Termogravimetria}

As análises termogravimétricas foram realizadas em uma termobalança Mettler Toledo, modelo TGA/SDTA-851, com variação de temperatura de 30 a $600{ }^{\circ} \mathrm{C}$ e razão de aquecimento de $10{ }^{\circ} \mathrm{C} \mathrm{min}{ }^{-1}$ sob atmosfera de hélio, com vazão de $25 \mathrm{~mL} \mathrm{~min}^{-1}$ utilizando cadinho de alumina de $900 \mu \mathrm{L}$ e massa de amostra de aproximadamente $60 \mathrm{mg}$. Na pesagem de padrões utilizou-se massa mais reduzida, aproximadamente $10 \mathrm{mg}$. A obtenção das derivadas das curvas de termogravimetria (DTG) permitiu avaliar os eventos de perdas de massa característicos de cada amostra analisada para a razão de aquecimento programada.

Foram selecionadas as seguintes amostras referentes ao produtor X: biodiesel de sebo bovino fresco, resíduo sólido filtrado das amostras X.1.A, X.2.A, X.3.A, X.6.A e X.12.A, correspondentes à simulação de verão (condição A) nos meses de armazenamento avaliados (1, 2, 3, 6 e 12 meses) e resíduo sólido filtrado das amostras X.1.B, X.1.C e X.1.D, correspondentes à simulação das diferentes condições e no tempo de 1 mês de armazenamento; referentes ao produtor Z: biodiesel de sebo bovino fresco, resíduo sólido filtrado das amostras Z.1.A, Z.2.A, Z.3.A, Z.6.A e Z.12.A, correspondentes à simulação de verão (condição A) nos meses de armazenamento avaliados (1, 2, 3, 6 e 12 meses) e resíduo sólido filtrado das amostras Z.1.B, Z.1.C e Z.1.D, correspondentes à simulação das diferentes condições e no tempo de 1 mês de armazenamento e padrões de monopalmitina e monoestearina.

\section{RESULTADOS E DISCUSSÃO}

\section{Avaliação dos teores de glicerídeos no biodiesel de sebo bovino}

No monitoramento das amostras de biodiesel de sebo bovino durante os 12 meses de armazenamento, verificou-se o mesmo comportamento nas concentrações de mono-, di- e triglicerídeos em ambos os produtores X e Z. A Figura 1 apresenta a evolução dos teores de glicerídeos correspondentes ao produtor X. A Figura $1 \mathrm{~S}$ (material suplementar) mostra a evolução dos teores de glicerídeos para o produtor $\mathrm{Z}$.

Verificou-se que os teores de mono-, di- e triglicerídeos no biodiesel de sebo bovino, praticamente, não se alteraram no período de 1 a 12 meses de armazenamento, independentemente da condição de armazenamento estudada, com exceção do B100 fresco. A manutenção dos mesmos níveis de teores de glicerídeos sugere estabilidade química destas espécies solubilizadas no biodiesel, mesmo após a formação do resíduo sólido, cuja precipitação pode ocorrer em estágio curto, não alterando as referidas concentrações no sobrenadante.

Cada produtor apresentou perfil característico de teores de mono-, di- e triglicerídeos no biodiesel de sebo bovino fresco, a saber, 0,55; 0,06 e $0,49 \%$ em massa (produtor X) e 0,56; 0,14 e 0,20\% em massa (produtor Z), respectivamente.

Os teores médios de mono-, di- e triglicerídeos, considerando-se todas as condições (A, B, C, D e E) e tempos de armazenamento ( 1 a 12 meses), foram 0,$51 ; 0,08$ e $0,51 \%$ em massa (produtor X) e 0,45; 0,16 e $0,46 \%$ em massa (produtor $Z$ ), respectivamente, ou seja, a soma dos teores de mono-, di- e triglicerídeos é de cerca de $1 \%$ em massa para ambos os produtores.

Comparando-se os teores de glicerídeos do B100 fresco e médios, verificou-se que houve redução de monoglicerídeos e aumento de (a)

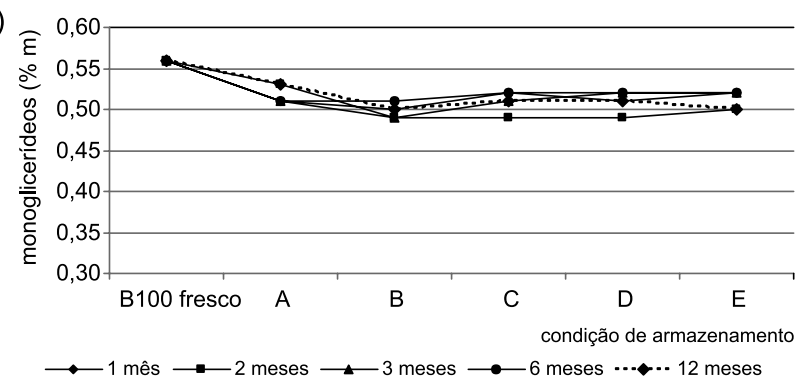

(b)

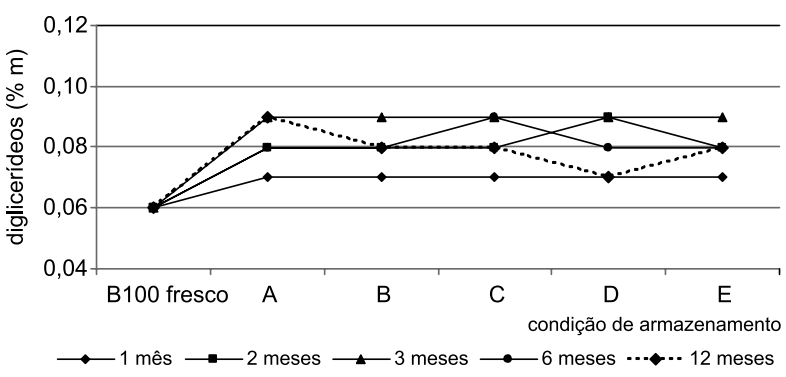

(c)

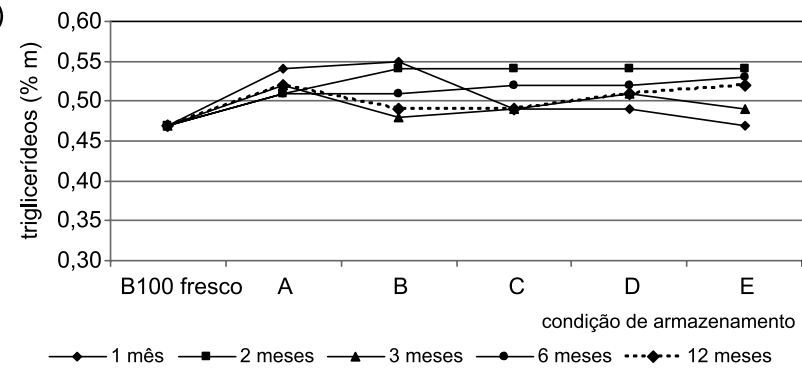

Figura 1. Curvas de teor de (a) monoglicerídeos, (b) diglicerídeos e (c) triglicerídeos do biodiesel de sebo bovino do produtor $X$

di- e triglicerídeos no biodiesel de sebo bovino, demonstrando que os monoglicerídeos precipitaram mais que os di- e triglicerídeos, sendo que este equilíbrio permaneceu estável por todo o tempo de armazenamento estudado.

\section{Natureza do resíduo sólido formado no biodiesel de sebo bovino}

Os resultados por CG-DIC da composição dos resíduos sólidos filtrados provenientes dos produtores $\mathrm{X}$ e $\mathrm{Z}$ foram parecidos. A Tabela 2 apresenta os percentuais de monoglicerídeos, diferenciados por monopalmitina, monoestearina e outros insaturados, analisados por CG-DIC, encontrados nos resíduos sólidos obtidos das amostras X.1.A, X.3.A e X.12.A, referentes ao produtor X, e amostras Z.1.A, Z.3.A e Z.12.A, referentes ao produtor $Z$, na condição A (simulação de verão) com 1 mês de armazenamento, respectivamente. O perfil cromatográfico dos padrões de referência utilizados, monopalmitina e monoestearina, do biodiesel de sebo bovino e dos resíduos sólidos analisados podem ser observados nas Figuras $2 \mathrm{~S}$ (produtor $\mathrm{X}$ ) e $3 \mathrm{~S}$ (produtor Z), no material suplementar.

A análise dos resultados por CG-DIC demonstrou que o resíduo sólido é constituído preponderantemente pelos monoglicerídeos saturados monopalmitina e monoestearina, cerca de 50\% da massa bruta, e pequena quantidade de outros monoglicerídeos insaturados ( $<0,4 \%$ massa), além do biodiesel residual não evaporado, massa restante, independente do produtor e do tempo de armazenamento. A quantidade de monoestearina foi superior cerca de $9 \%$, em média, da quantidade de monopalmitina constituinte do resíduo 
Tabela 2. Percentuais de monoglicerídeos presentes nos resíduos sólidos, analisados por CG-DIC

\begin{tabular}{cccc}
\hline \multicolumn{4}{c}{ Produtor X - teor de monoglicerídeos (\% massa)* } \\
\hline Resíduo & Monopalmitina & Monoestearina & $\begin{array}{c}\text { Monoglicerídeos } \\
\text { insaturados }\end{array}$ \\
\hline X.1.A & 20,3 & 23,1 & 0,17 \\
X.3.A & 25,7 & 27,8 & 0,27 \\
X.12.A & 24,4 & 26,2 & 0,34 \\
\hline \multicolumn{4}{c}{ Produtor Z-teor de monoglicerídeos (\% massa)* } \\
\hline Z.1.A & 23,2 & 26,1 & 0,29 \\
Z.3.A & 20,0 & 21,7 & 0,23 \\
Z.12.A & 20,0 & 21,8 & 0,23 \\
\hline
\end{tabular}

* Teores de monoglicerídeos em relação à massa total. O restante das amostras contém ésteres, diglicerídeos e triglicerídeos (estes em quantidades de traço).

sólido. Não foram detectados di- e triglicerídeos no precipitado. A proximidade dos percentuais de monoglicerídeos na composição do resíduo sólido nos diferentes tempos de armazenamento sugere que a precipitação ocorre em estágio curto e irreversível, pela nucleação e aglomeração destas moléculas no biodiesel de sebo bovino, com preponderância do ciclo de precipitação no primeiro mês de armazenamento.

Uma possível explicação para a composição majoritária de monopalmitina e monoestearina nos precipitados de biodiesel de sebo bovino seria devida à estrutura química dos monoglicerídeos saturados (Figura 4S, material suplementar), pois é a classe de glicerídeos presentes no biodiesel com maior caráter polar e apolar na mesma molécula. Essa característica favoreceria a aglutinação de moléculas semelhantes, mais do que em qualquer outro componente do biodiesel. O mesmo não é esperado ocorrer com os monoglicerídeos insaturados. ${ }^{16}$

Vale lembrar que o objetivo do trabalho foi identificar a natureza do resíduo sólido formado no biodiesel de sebo bovino. Não foi intenção estender um balanço de massa em função da metodologia utilizada para separação do resíduo sobrenadante (biodiesel), ou seja, uso de papel de filtro sob vácuo, o que possibilita vazamento de traços de resíduo. Além disso, a secagem do resíduo foi em temperatura ambiente, para evitar perdas do mesmo (composto majoritariamente de monoglicerídeos) por decomposição. Desta forma, o resíduo ficou impregnado com traços de biodiesel, o que poderia mascarar os resultados de um balanço de massa.

\section{Análise termogravimétrica}

As curvas TG/DTG possibilitaram a verificação das temperaturas de perdas de massa dos resíduos sólidos das amostras de biodiesel de sebo bovino provenientes dos produtores $\mathrm{X}$ e Z , representativas do início até o final do estudo. Os materiais foram aquecidos na faixa de temperatura de 30 a $600{ }^{\circ} \mathrm{C}$ em razão de aquecimento de $10{ }^{\circ} \mathrm{C}$ $\mathrm{min}^{-1}$, sob atmosfera de hélio, com vazão de $25 \mathrm{~mL} \mathrm{~min}{ }^{-1}$. A Figura 2 apresenta as curvas DTG dos resíduos sólidos e do biodiesel de sebo bovino do produtor $\mathrm{X}$. Foram observados os mesmos comportamentos de decomposição térmica das correspondentes amostras provenientes do produtor Z, cujas figuras foram omitidas neste trabalho. A Figura 3 apresenta as curvas DTG dos resíduos X.1.A e Z.1.A (condição A e 1 mês de armazenamento) comparadas com as dos padrões monopalmitina e monoestearina.

Os perfis das curvas termogravimétricas dos resíduos sólidos demonstraram o mesmo comportamento de perda de massa, independente do produtor, da condição e do tempo de armazenamento. Foram
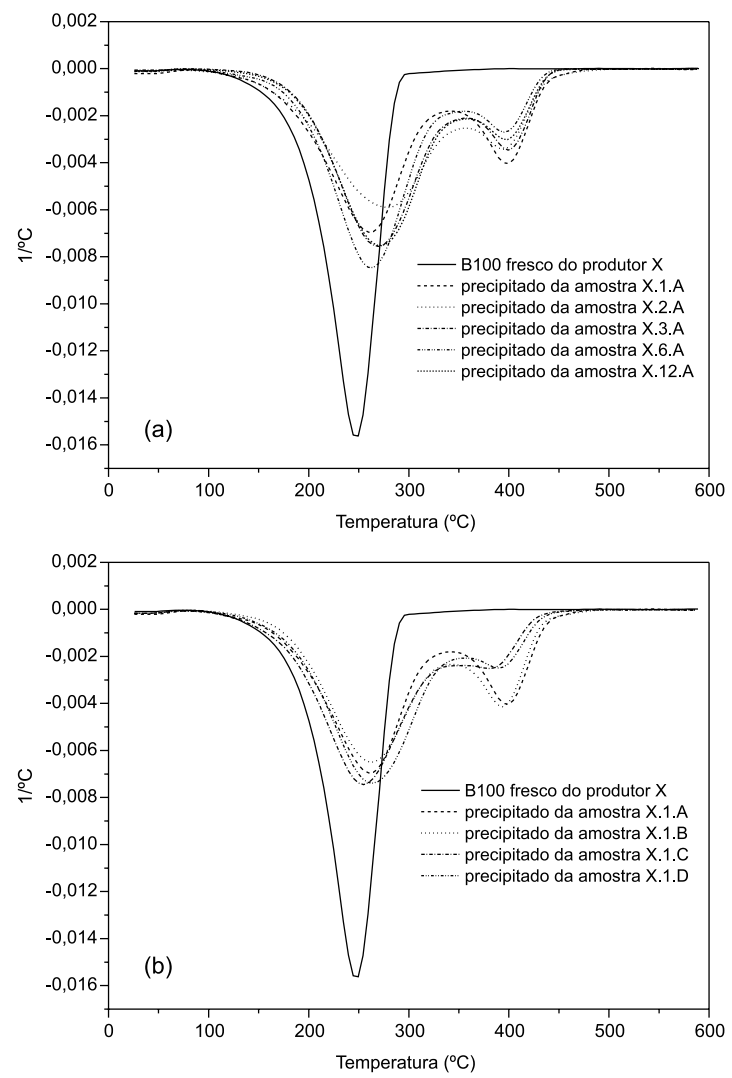

Figura 2. Sobreposição das curvas DTG do biodiesel de sebo bovino e dos resíduos sólidos filtrados das amostras provenientes do produtor X: (a) referentes à simulação de verão (condição A) nos meses de armazenamento avaliados (1, 2, 3, 6 e 12 meses) e (b) referentes à simulação das diferentes condições $(A, B, C$ e $D)$ e tempo de 1 mês de armazenamento

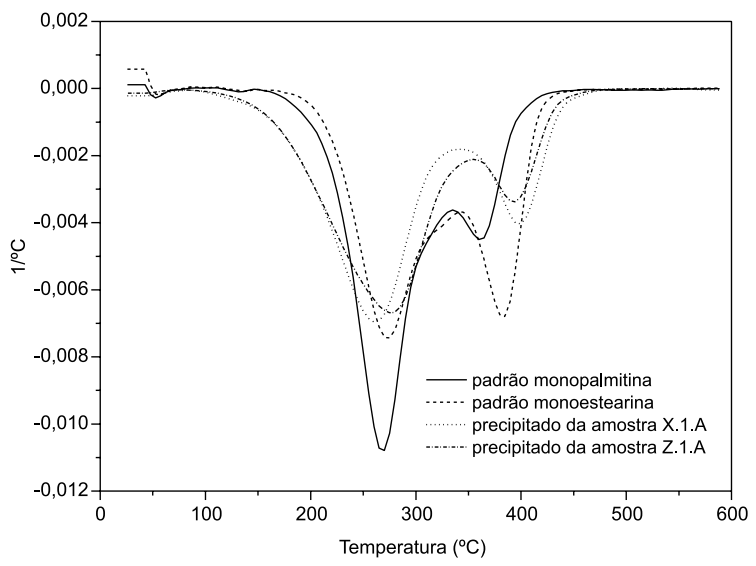

Figura 3. Sobreposição das curvas DTG dos padrões monopalmitina, monoestearina e resíduos X.1.A e Z.1.A

observadas 2 etapas com perdas de massa nas análises dos resíduos sólidos e dos padrões monopalmitina e monoestearina e apenas 1 etapa com perda de massa referente ao biodiesel de sebo bovino, de ambos os produtores $\mathrm{X}$ e $\mathrm{Z}$, nas condições de aquecimento programadas.

Os percentuais de perdas de massa e das massas residuais obtidas estão apresentados na Tabela 3.

A análise dos resultados demonstrou que os resíduos sólidos possuem as mesmas composições químicas por apresentarem perfis termogravimétricos similares, independente do produtor, do tempo e da condição de armazenamento. Os resíduos sólidos apresentaram, também, perdas de massa dentro das faixas de temperaturas dos 
Tabela 3. Faixas de temperatura e correspondentes perdas de massa das TG/ DTG das amostras analisadas

\begin{tabular}{|c|c|c|c|c|c|}
\hline \multirow{3}{*}{ Amostra analisada } & \multirow{2}{*}{\multicolumn{2}{|c|}{$\frac{\text { Temperatura }\left({ }^{\circ} \mathrm{C}\right)}{\text { Etapa }}$}} & \multirow{2}{*}{\multicolumn{2}{|c|}{$\begin{array}{c}\text { Perda de massa }(\%) \\
\text { Etapa }\end{array}$}} & \multirow{3}{*}{$\begin{array}{l}\text { Massa } \\
\text { residual } \\
\quad(\%)\end{array}$} \\
\hline & & & & & \\
\hline & 1 & 2 & 1 & 2 & \\
\hline B100 fresco & $73-370$ & - & 99,2 & - & 0,8 \\
\hline resíduo X.1.A & $77-341$ & $341-469$ & 71,9 & 26,0 & 2,1 \\
\hline resíduo X.2.A & $68-356$ & $356-473$ & 73,0 & 25,9 & 1,1 \\
\hline resíduo X.3.A & $77-356$ & $356-463$ & 75,7 & 22,9 & 1,4 \\
\hline resíduo X.6.A & $77-356$ & $356-466$ & 83,8 & 15,5 & 0,7 \\
\hline resíduo X.12.A & $83-356$ & $356-461$ & 81,0 & 17,9 & 1,1 \\
\hline resíduo X.1.B & $80-345$ & $345-473$ & 71,5 & 26,6 & 1,9 \\
\hline resíduo X.1.C & $76-357$ & $357-474$ & 85,8 & 13,2 & 1,0 \\
\hline resíduo X.1.D & $73-354$ & $354-460$ & 83,4 & 15,2 & 1,4 \\
\hline resíduo Z.1.A & $79-354$ & $354-483$ & 79,0 & 20,0 & 1,0 \\
\hline resíduo Z.2.A & $90-340$ & $340-439$ & 74,7 & 24,7 & 0,6 \\
\hline resíduo Z.3.A & $73-337$ & $337-441$ & 77,0 & 22,6 & 0,4 \\
\hline resíduo Z.6.A & $76-335$ & $335-452$ & 72,0 & 26,6 & 1,4 \\
\hline resíduo Z.12.A & $90-332$ & $332-450$ & 70,9 & 28,0 & 1,1 \\
\hline resíduo Z.1.B & $77-329$ & $329-482$ & 67,9 & 31,5 & 0,6 \\
\hline resíduo Z.1.C & $79-350$ & $350-441$ & 73,6 & 24,7 & 1,8 \\
\hline resíduo Z.1.D & $79-342$ & $342-460$ & 72,3 & 26,3 & 1,4 \\
\hline monopalmitina & $151-335$ & $335-450$ & 77,1 & 22,1 & 0,8 \\
\hline monoestearina & $172-345$ & $345-464$ & 64,0 & 34,1 & 1,9 \\
\hline
\end{tabular}

padrões monopalmitina e monoestearina, evidenciando a presença predominante destes monoglicerídeos em suas composições.

Os valores de perdas de massa dos resíduos sólidos dos produtores X e Z (Tabela 3) indicaram que o resíduo sólido na etapa 1 apresentou maior quantidade de material estável até $357{ }^{\circ} \mathrm{C}$, em torno de $76 \%$ da massa total, enquanto que a etapa 2 deteve em torno de $23 \%$ de material estável a temperaturas de até $483{ }^{\circ} \mathrm{C}$, permanecendo aproximadamente $1 \%$ de massa residual. Este perfil se assemelha aos dos padrões utilizados. De acordo com Frömming e Szejtli, o perfil termogravimétrico da monopalmitina é caracterizado por 2 etapas com perdas de massa, sendo a primeira devida à volatilização do monoglicerídeo e a segunda, à decomposição de complexo formado pelas moléculas da monopalmitina quando submetidas a altas temperaturas..$^{20}$ Por analogia, a ocorrência de 2 etapas de perdas de massa dos resíduos sólidos corresponde, primeiramente, à volatilização/evaporação dos compostos preponderantes, monopalmitina e monoestearina, além do residual de biodiesel de sebo bovino não evaporado. A etapa 2 corresponde à pirólise de produtos originados da complexação e polimerização dos constituintes do resíduo sólido, caracterizados por possuírem cadeias carbônicas mais pesadas e grupamentos acila e carboxila provenientes do biodiesel. O percentual de massa residual obtido refere-se ao coque oriundo do resíduo submetido a altas temperaturas.

A única etapa de perda de massa observada nas amostras do biodiesel de sebo bovino é característica da volatilização/evaporação dos triglicerídeos e ésteres metílicos, correspondendo a 99,2\%, para o produtor X, e $98,9 \%$, para o produtor Z, de material estável a temperaturas de até $370{ }^{\circ} \mathrm{C} .{ }^{21}$ As massas residuais obtidas no final das análises termogravimétricas foram praticamente iguais, $0,8 \%$ (produtor $\mathrm{X}$ ) e $1,1 \%$ (produtor $\mathrm{Z}$ ), indicando amostras com composições similares.
Os resultados de termogravimetria corroboraram com os obtidos por CG-DIC acerca do resíduo sólido de biodiesel de sebo bovino ser composto preponderantemente por monopalmitina e monoestearina.

\section{CONCLUSÕES}

A análise por CG-DIC verificou que houve redução de monoglicerídeos e aumento de diglicerídeos e triglicerídeos no biodiesel de sebo bovino após a formação do resíduo sólido, sendo mantidos, praticamente, estes mesmos teores nos 12 meses de armazenamento. Tal fato demonstra que a natureza do resíduo é proveniente da nucleação e aglomeração de monoglicerídeos saturados, a partir de uma névoa fina, majoritariamente, no primeiro mês de armazenamento.

$\mathrm{Na}$ decomposição térmica, o resíduo sólido apresentou duas etapas de perdas de massa: a primeira, principal, referente à volatilização dos monoglicerídeos saturados monopalmitina e monoestearina, e a segunda correspondente à pirólise de produtos originados da complexação e polimerização dos constituintes do resíduo sólido. O biodiesel de sebo bovino apresentou uma única etapa de perda de massa referente à decomposição de triglicerídeos e ésteres metílicos.

Resultados de CG-DIC e TG demonstraram que o resíduo sólido de biodiesel de sebo bovino é constituído majoritariamente por monoestearina e monopalmitina. Não foram detectados diglicerídeos e triglicerídeos no resíduo sólido.

\section{MATERIAL SUPLEMENTAR}

Está disponível em http://quimicanova.sbq.org.br, em arquivo pdf, com acesso livre. Nele encontram-se a evolução dos teores de glicerídeos correspondentes ao biodiesel de sebo bovino do produtor Z (Figura 1S), o perfil cromatográfico dos padrões de referência monopalmitina e monoestearina, do biodiesel de sebo e dos resíduos sólidos das amostras X.1.A, X.3.A e X.12.A, referentes ao produtor X (Figura 2S) e amostras Z.1.A, Z.3.A e Z.12.A, referentes ao produtor Z (Figura 3S). A Figura 4 S apresenta a estrutura química de monopalmitina (a) e monoestearina (b).

\section{REFERÊNCIAS}

1. ANP - Agência Nacional do Petróleo, Gás Natural e Biocombustíveis; Resolução No 7 de 19/3/2008; Diário Oficial da União de 20/3/2008.

2. Fangrui, M.; Milford, A. H.; Bioresour. Technol. 1999, 70, 1.

3. Tang, H.; Salley, S. O.; Simon, K. Y.; Fuel 2008, 87, 3006.

4. Connatser, R. M.; Kass, M. D.; Lewis, S. A.; US pat. 12/820,177 2010.

5. Tang, H.; Guzman, R. C.; Salley, S. O.; J. Am. Oil Chem. Soc. 2008, 85, 1173.

6. van Hoed, V.; Zyakina, N.; de Greyt, W.; Maes, J.; Verhe, R.; Demestere, K.; J. Am. Oil Chem. Soc 2008, 85, 701.

7. Jonker, D.; Glatz, J. F. C.; Homan, C.; Posthumus, M. A.; Vanderhoek, G. D.; Katan, M. B.; Nutr. Rep. Int. 1985, 32, 943.

8. Hudson, E. D.; Parrish, C. C.; Helleur, R. J.; Mar. Chem. 2001, 76, 253.

9. Lee, I.; Pfalzgraf, L. M.; Poppe, G. B.; Powers, E.; Haines, T.; Biodiesel Magazine 2007, 4, 105.

10. Pieber, B.; Schober, S.; Goebl, C.; Mittelbach, M.; J. Chromatogr., A 2010, 1217,6555 .

11. Moreau, R. A.; Scott, K. M.; Haas, M. J.; J. Am. Oil Chem. Soc. 2008, 85,761 .

12. Brasil; Lei $\mathrm{N}^{\circ} 11.097$ de 13/1/2005; Diário Oficial da União de $14 / 1 / 2005$.

13. http://www.biodieselbr.com/noticias/em-foco/brasil-segundo-maiorprodutor-biodiesel-mundo-2010-ministro-23-10-09.htm, acessada em Maio 2011. 
14. ANP - Agência Nacional do Petróleo, Gás Natural e Biocombustíveis; Informe de Biocombustíveis 2009, $4^{\mathrm{a}}$ ed.

15. Moura, K. R. M.; Silva, F. C.; Brandão, K. S. R.; Souza, A. G.; Anais do $1^{\circ}$ Congresso da Rede Brasileira de Tecnologia do Biodiesel, Brasília, Brasil, 2006.

16. Pacheco, W. O.; Vinhado, F. S.; Resumos do III Congresso da Rede Brasileira de Tecnologia de Biodiesel, Brasília, Brasil, 2009.

17. ASTM - American Society for Testing and Materials; Standard Test Method for Determination of Total Monogliceride, Total Digliceride,
Total Trigliceride, and Free and Total Glycerin in B-100 Biodiesel Methyl Esters by Gas Chromatography, ASTM D 6584, 2010.

18. Goh, E. M.; Timms, R. E.; J. Am. Oil Chem. Soc. 1985, 62, 730

19. Plank, C.; Lorbeer, E.; J. Chromatogr., A 1995, 697, 461.

20. Frömming, K. H.; Szejtli, J.; Cyclodextrins in pharmacy, Topics in inclusion science, Kluwer Academic Publisher: Dordrecht, The Netherlands, 1993.

21. Santos, A. G. D.; Araujo, A. S.; Caldeira, V. P. S.; Fernandes Jr., V. J.; Souza, L. D.; Barros, A. D.; Thermochim. Acta 2010, 506, 57 


\section{CARACTERIZAÇÃO DE RESÍDUO SÓLIDO FORMADO EM BIODIESEL DE SEBO BOVINO}

Valter José Fernandes Júnior e Antonio de Sousa Araujo

Instituto de Química, Universidade Federal do Rio Grande do Norte, Campus Universitário, s/n, 59078-970 Natal - RN, Brasil Fábio da Silva Vinhado e Paulo Roberto Pivesso*

Centro de Pesquisas e Análises Tecnológicas, Agência Nacional do Petróleo, Gás Natural e Biocombustíveis, SGAN 603, Módulo H/J, 70830-902 Brasília - DF, Brasil

A Figura 1S apresenta a evolução dos teores de glicerídeos correspondentes ao biodiesel de sebo bovino do produtor $\mathrm{Z}$.

O perfil cromatográfico dos padrões de referência monopalmitina e monoestearina, do biodiesel de sebo e dos resíduos sólidos das amostras X.1.A, X.3.A e X.12.A, referentes ao produtor X é apresentado na Figura $2 \mathrm{~S}$.

As amostras Z.1.A, Z.3.A e Z.12.A, referentes ao produtor Z, estão representadas na Figura 3S.

A análise dos resultados demonstrou que os tempos de retenção (TR) referentes aos picos cromatográficos da monopalmitina e da monoestearina foram 16,25 e 17,74 min, respectivamente, para todos os resíduos analisados provenientes do produtor $\mathrm{X}$ e do produtor $\mathrm{Z}$, além do biodiesel de sebo bovino. Estes TR foram os mesmos obtidos pelos padrões monopalmitina e monoestearina utilizados como referências, evidenciando preponderância destes compostos na massa dos resíduos sólidos. Verificou-se, também, que outros monoglicerídeos insaturados presentes no biodiesel de sebo bovino, com TR típico de 17,58 min para a mono-oleína, não foram encontrados nos resíduos sólidos, inclusive, também, diglicerídeos e triglicerídeos (TR 21,03 a 21,25 e 22,16 a 22,5 min, respectivamente) observados no biodiesel de sebo bovino. A Figura $4 \mathrm{~S}$ apresenta a estrutura química de monopalmitina (a) e monoestearina (b).

(a)

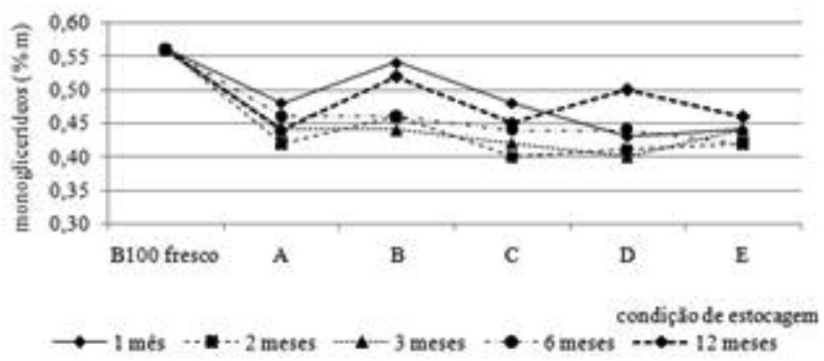

(b)

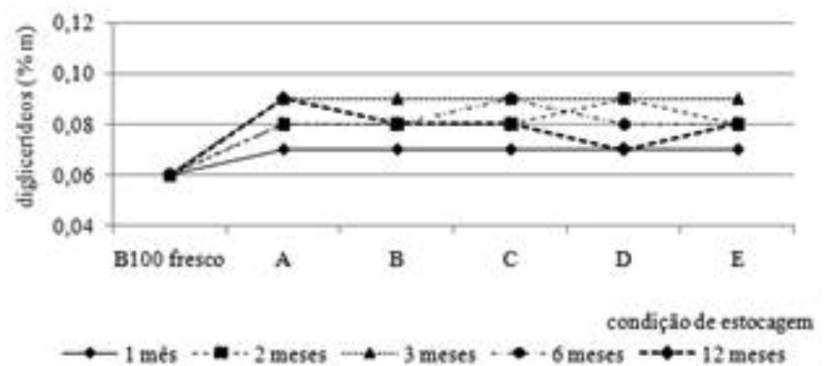

(c)

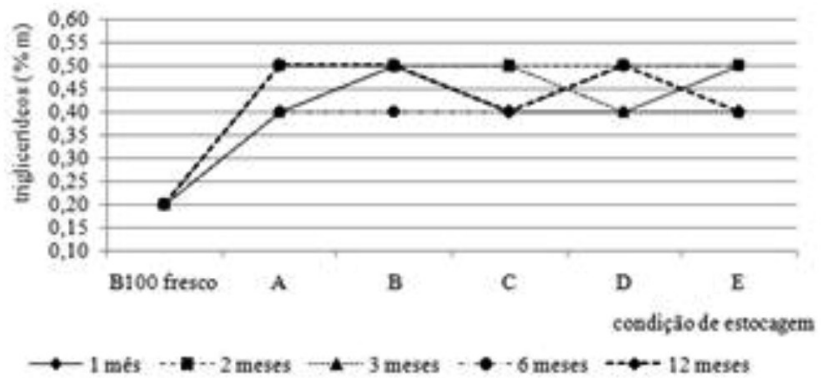

Figura 1S. Curvas de teor de (a) monoglicerídeos, (b) diglicerídeos e (c) triglicerídeos do biodiesel de sebo bovino do produtor Z 
(a)

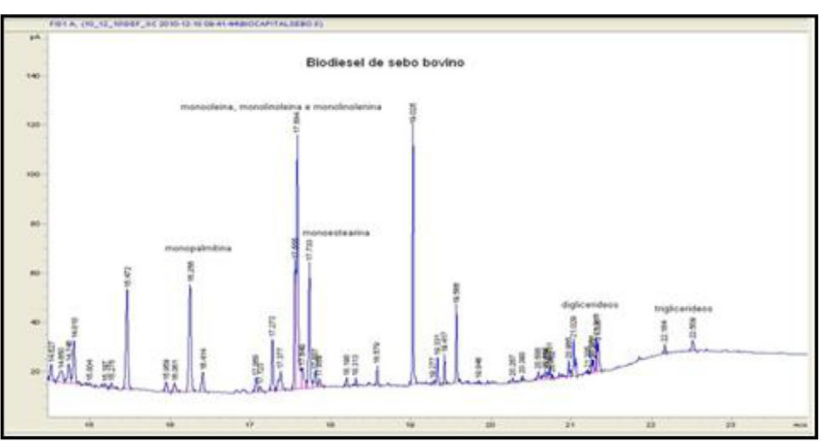

(c)

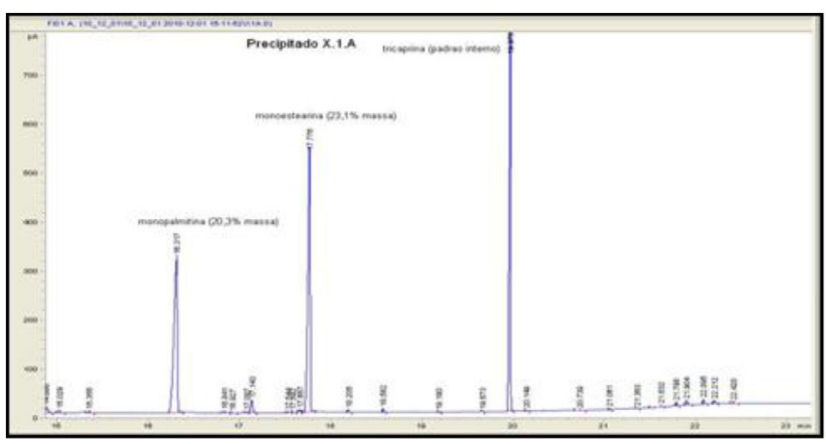

(b)

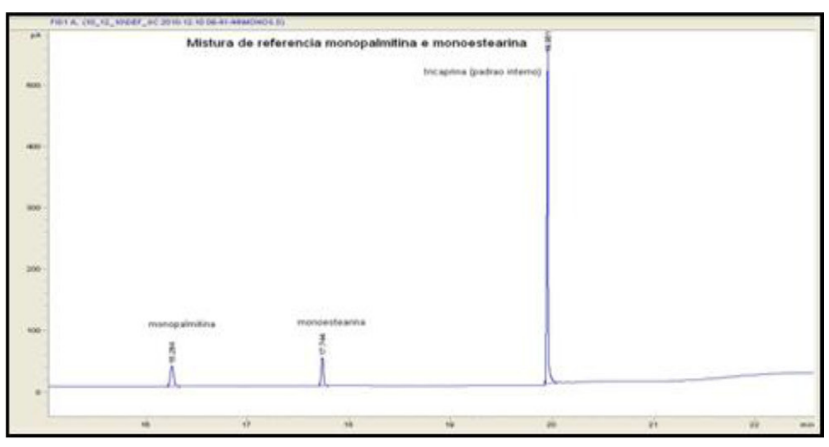

(d)

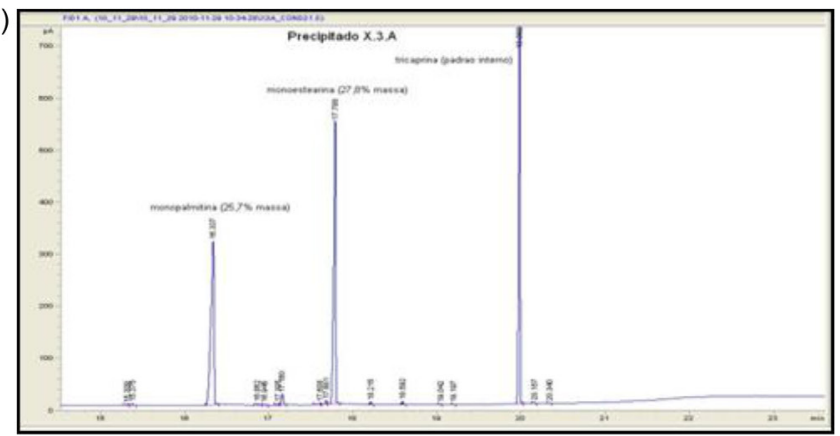

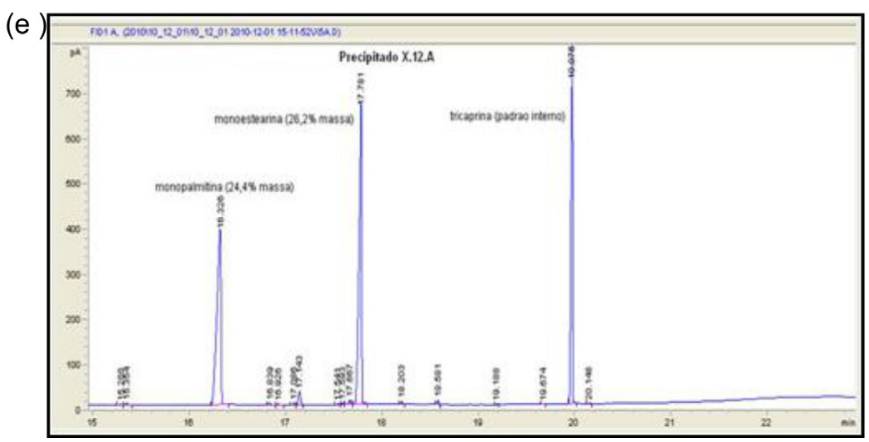

Figura 2S. Cromatogramas CG-DIC das amostras: (a) biodiesel de sebo bovino, (b) padrões monopalmitina e monoestearina e resíduos filtrados do produtor $X$, referentes à simulação de verão (condição A) nos períodos de armazenamento de 1, 3 e 12 meses, precipitados, (c) X.1.A, (d) X.3.A e (e) X.12.A 
(a)

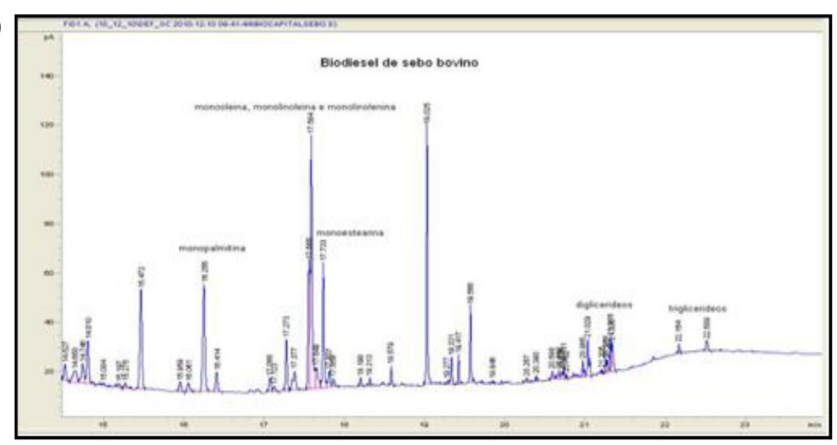

(c)

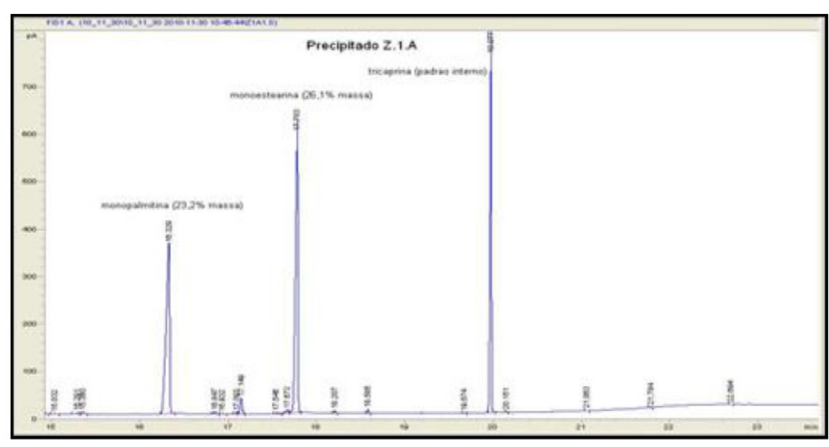

(b)

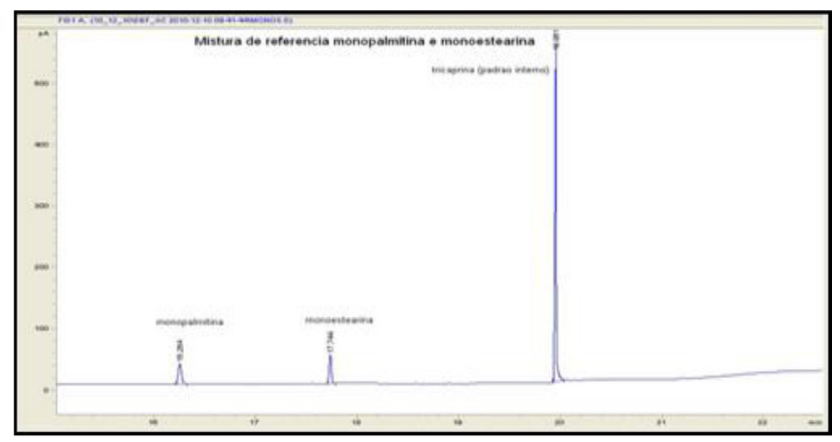

(d)

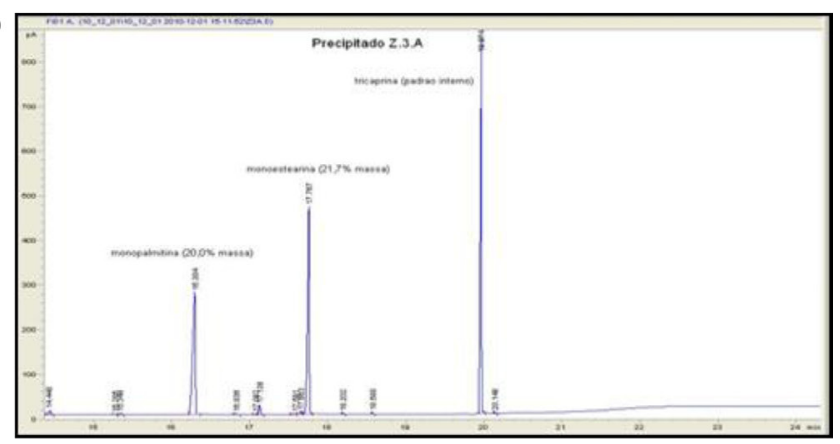

(e )

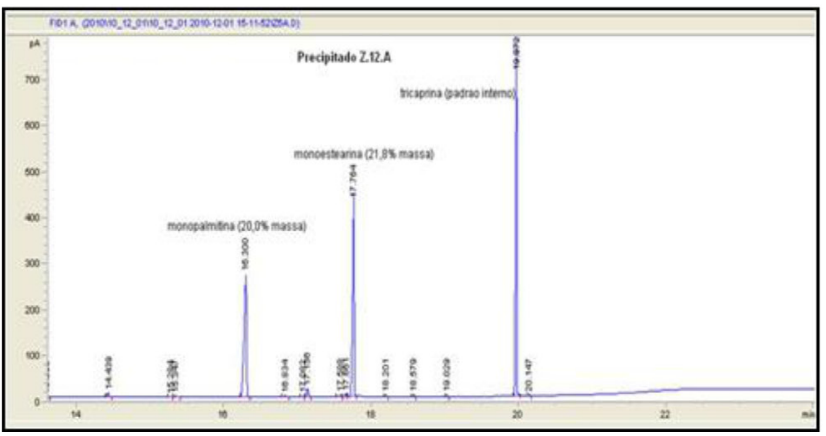

Figura 3S. Cromatogramas CG-DIC das amostras: (a) biodiesel de sebo bovino, (b) padrões monopalmitina e monoestearina e resíduos filtrados do produtor $Z$, referentes à simulação de verão (condição A) nos períodos de armazenamento de 1, 3 e 12 meses, precipitados, (c) Z.1.A, (d) Z.3.A e (e) Z.12.A
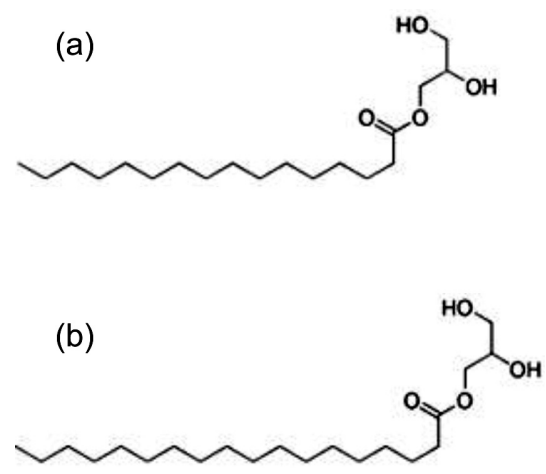

Figura 4S. Representação estrutural de monopalmitina (a) e monoestearina (b) 